\title{
A Modified Formal Theory on Semi-Relativistic Effects during Electron Emission from Metallic Surfaces upon the Impact of High Energy Particles
}

\author{
S. Dhar ${ }^{*}$, M. G. Hafez, A. Saha \\ Department of Mathematics, Chittagong University of Engineering and Technology, Chittagong, Bangladesh \\ Email: "sdhar@cuet.ac.bd, *sdhar03@yahoo.com
}

Received July 29, 2013; revised August 29, 2013; accepted September 5, 2013

Copyright (C) 2013 S. Dhar et al. This is an open access article distributed under the Creative Commons Attribution License, which permits unrestricted use, distribution, and reproduction in any medium, provided the original work is properly cited.

\begin{abstract}
In this paper we introduce a formal theory on unveiling relativistic effects during electron emission from clean metallic surfaces upon high charged particle impact using a Jellium-type wave function including suitable spinors. In addition image charge final state electron surface interactions have been initiated in the relativistic region as well as the scattering of the projectile from the multi-center bulk potential. Finally, a semi-relativistic condition is considered in place of the ionization mechanism of scattering from an aluminium semi-infinite solid target by non-relativistic electrons to determine multiple differential cross-section.
\end{abstract}

Keywords: Ionization; Cross-Section; Relativistic; Electron; Scattering

\section{Introduction}

In the last three decades significant progress has been made in understanding the atomic physics of electronatom ionization both theoretically and experimentally for relativistic energies [1-14] as well as for non-relativistic energies [15-26]. Capabilities of different theoretical models in reproducing various features of experimental results have been widely tested for different kinematical conditions. Study of energy spectrum of ejected electrons could be very interesting. The complexity of this reaction is already revealed in the simplest case of low-energy electron impact ionization of atomic hydrogen where final state interactions of escaping particles strongly modify the observed electron spectra $[16,21]$. Hence a realistic approximate eigenfunction of the non-separable three body Hamiltonian is essential. Generally, a theoretical description of this process from solid targets has to deal with various aspects of the beam-solids interactions. A relativistic charge particle impinging on a metallic target, which is the study of this work, leads to charge-density fluctuation of the solid. Asymptotically, this causes an image-charge distribution of the incoming and outgoing particles [22]. Moreover, the motion of the various electrons is periodically distributed by the interaction with

\footnotetext{
"Corresponding author.
}

the bulk potential $[24,25]$. The aim of this paper is to investigate the energy spectrum of electron ejection from clean metalic surfaces upon higher charged particle impact for relativistic energies $[10,12,23]$. The present study of the formal theory is the assumption that the degrees of freedom of the projectile can be decoupled from those of the target. This is justified, since we assumed the momenta of the incoming and outgoing electron to be considerably larger than the Fermi momentum. To obtain the analytical theory that can be analyzed, a Jellium wave function [23] with suitable spinors [12] of the initially bound electron is assumed. Asymptotic image-charge distribution of the Vacuum electron is taken into account. As well as the binary collision of the projectile with the bound electron, the scattering of the incident particle from the multicenter bulk potential is treated in the kinematic approximation [25,26]. A screened Coulomb muffin-tin bulk potential is adopted. Recently using the multiple scattering theory [17] multiplied with some spinors has calculated the energy spectrum of scattered electrons in $\mathrm{k}$-shell ionization of medium heavy atoms by relativistic electrons [10-12] which agree nicely with experiments. In the present study we developed the theory of relativistic $(\mathrm{e}, 2 \mathrm{e})$ scattering mechanism process using a Jellium wave function [23] of the undistributed surface in the 
initial state. In addition, image-charge final state electron surface interactions have been included. Moreover, the scattering of the projectile from the multicentre bulk potential is considered. The proposed theory will provide scattering cross-sections results of different kinematical conditions. Hence we introduced a modified theory on semi-relativistic effects during electron emission from aluminium surfaces upon the impact of high energy particles.

\section{Theory}

In this theory, we consider an atomic scattering system consisting of a projectile with nuclear change $Z_{p}$ and mass $m_{p}$ being inelastically scattered from a clean metallic semi-infinite solid ejecting one electron into the vacuum level of the solid. Then the total Hamiltonian of the projectile-solid system is

$$
H=H_{p}+H_{e}+W_{p e}
$$

where $H_{e}$ is the Hamiltonian of the simi-infinite solid in the absence of the projectile, $W_{p e}$ is the projectileejected electron interaction, and $H_{p}$ represents the projectile-crystal interaction and contains the plasmon and phonon modes and their interaction with electron. The initial and final state boundary conditions are specified by eigenstates $|i\rangle,|f\rangle$ of asymptotically unperturbed initial and final channel Hamiltonian $H_{i}$ and $H_{f}$ respectively, i.e.,

$$
\begin{gathered}
\left(H_{i}-E_{i}\right)|i\rangle=0 \\
\left(H_{f}-E_{f}\right)|f\rangle=0,
\end{gathered}
$$

where $E_{i}, E_{f}$ are the corresponding asymptotic eigen energies. The transition amplitude $T$ for the scattering system from initial state $|i\rangle$, to the final state $|f\rangle$ is determined by the prior form

$$
T=\left\langle\psi^{-}\left|V_{i}\right| i\right\rangle
$$

or the post form

$$
T=\left\langle f\left|V_{f}\right| \psi^{+}\right\rangle,
$$

where $\left|\psi^{+}\right\rangle$and $\left|\psi^{-}\right\rangle$are the eigensates of the total Hamiltonian $\mathrm{H}$ according to the state $|i\rangle$ and $|f\rangle$ respectively. From Equations (4) and (5) the perturbations potential $V_{i}$ and $V_{f}$ are given by

$$
\begin{gathered}
V_{i}=H-H_{i} \\
V_{f}=H-H_{f} .
\end{gathered}
$$

The eigenstates $\left|\psi^{-}\right\rangle$of $\mathrm{H}$ can be written as

$$
\left|\psi^{-}\right\rangle=\Omega^{-}|f\rangle
$$

$$
\Omega^{-}=1+G^{-} V_{f}
$$

where $G^{-}$is the Green operator of the total Hamiltonian $H$. Combining Equations (9), (8) and (4), the $T$ matrix element may be written as

$$
T=T^{\sin }+T^{\text {mult }},
$$

where

$$
\begin{gathered}
T^{\sin }=\left\langle f\left|V_{i}\right| i\right\rangle, \\
T^{\text {mult }}=\left\langle f\left|V_{f} G^{+} V_{i}\right| i\right\rangle .
\end{gathered}
$$

Here assuming $V_{i}$ to be a multiple-center potential, the first term of Equation (10), that is $T^{\sin }$ describes the transition of the system from state $|i\rangle$ to $|f\rangle$ due to a single scattering from each individual scattering center. One center and multicenter multiple scattering is contained in the matrix element $T^{\text {mult }}$ in Equations (10) and (12), since the Lippmann-Schwinger equation of the total Green operator leads to the expansion

$$
T^{\text {mult }}=\left\langle f\left|V_{f} G_{0}^{+} \sum_{j=0}^{\infty}\left[V G_{0}^{+}\right]^{j}\right| i\right\rangle,
$$

where $G_{0}^{+}$and $V$ are the full free propagator and the total potential of the projectile-solid compound. It should be noted here that the labels $\sin$ and mult of the amplitudes $T^{\text {sin }}$ and $T^{\text {mult }}$ refer to single and multiple scattering specifically from the potential $V_{i}$. The potential $V_{i}$ in the initial channel can be written as

$$
V_{i}=W_{p e}+W_{p}^{s}+W_{p}^{v a c} .
$$

The operator $W_{p}^{s}$ stands for the particle-bulk interaction and its explicit functional form is specified below. The potential $W_{p}^{v a c}$ is less prominent. Since it is an asymptotic (imagine charge) perturbation. For the asymptotic final-channel Hamiltonian [23] is given by

$$
H_{f}=k_{e}+k_{p}+W_{p}^{v a c}+W_{e}^{v a c}+W_{p e} .
$$

Here the kinetic-energy operator of the secondary electron is referred to by $k_{e}$, whereas $W_{e}^{v a c}$ amounts to the asymptotic final state interaction of this electron with semi-infinite solid. The choice Equation (15) leads to the final-channel distribution operator

$$
V_{f}=W_{p}^{s}+W_{e}^{s},
$$

where $W_{e}^{s}$ denotes the short-range interaction of the secondary electron with the surface. Upon substitution of Equation (14) into Equation (11), the matrix element $T^{\text {sin }}$ can be decomposed into the form

$$
T^{\sin }=T_{p e}^{\sin }+T_{p, s}^{\sin }+T_{p, v}^{\sin },
$$

where

$$
T_{p e}^{s i n}=\left\langle f\left|W_{p e}\right| i\right\rangle,
$$




$$
\begin{aligned}
T_{p, s}^{\sin } & =\left\langle f\left|W_{p}^{s}\right| i\right\rangle, \\
T_{p, v}^{\sin } & =\left\langle f\left|W_{p}^{v a c}\right| i\right\rangle .
\end{aligned}
$$

The amplitudes, given by Equations (18) and (19), provide the first-order approximation of the matrix element $T$. The term in Equation (20) is less prominent since $W_{p}^{v a c}$ is an asymptotic (image charge) perturbation. Hence it has no significant contribution to the $T^{\sin }$ term. So, the amplitude, given by Equation (20) is neglected here.

\subsection{Analytical Calculation of the Transition Amplitude Term $T_{p e}^{\text {sin }}$ in Relativistic Effects}

In a position-space representation the transition operator occurring in the Equation (18) has the form

$$
W_{p e}=-Z_{e f f} \alpha_{0} /\left|r_{p}-r_{e}\right|,
$$

where $\boldsymbol{r}_{p}$ and $\boldsymbol{r}_{e}$ are the position vectors of the projectile and ionized electron respectively. Also $\alpha_{0}$ is time structure constant and $Z_{\text {eff }}$ (effective nuclear charge) $=$ Nuclearcharge $\left(z_{p}\right)-0.3$. To avoid difficulties arising from the infinite range of coulomb interactions we introduce the cutoff potential [23]

$$
W_{p e}=\lim _{\eta_{1} \rightarrow 0^{+}} \frac{-Z_{e f f} \alpha_{0} \exp \left(-\eta_{1}\left|\boldsymbol{r}_{e}-\boldsymbol{r}_{p}\right|\right)}{\left|\boldsymbol{r}_{e}-\boldsymbol{r}_{p}\right|} .
$$

The eigenfunction of $H_{f}$ of Equation (15) at a given asymptotic energy $E_{f}$ [23] is readily deduced in semirelativistic form $[1,12]$ as

$$
\begin{aligned}
& \left\langle\boldsymbol{r}_{e}, \boldsymbol{r}_{p}, \sigma_{e}^{\prime}, \sigma_{p}^{\prime} \mid f\right\rangle \\
\equiv & \phi_{f}\left(\boldsymbol{r}_{e}, \boldsymbol{r}_{p}, \sigma_{e}^{\prime}, \sigma_{p}^{\prime}\right) \\
= & (2 \pi)^{-3} \exp \left[i \boldsymbol{k}_{p} \cdot \boldsymbol{r}_{p}+i \boldsymbol{k}_{e} \cdot \boldsymbol{r}_{e}-i \varphi\left(\boldsymbol{r}_{e}\right)\right] u_{\sigma_{p}^{\prime}}\left(\boldsymbol{k}_{e}\right) u_{\sigma_{e}^{\prime}}\left(\boldsymbol{k}_{e}\right) .
\end{aligned}
$$

In addition the Jelliun wave function [23] can be expressed in terms of reflection and transmission coefficient may be written as in semi-relativistic form $[1,12]$ as

$$
\begin{gathered}
\left\langle\boldsymbol{r}_{e}, \sigma_{p}, \sigma_{e} \mid \chi_{k}\right\rangle=\frac{\left(\alpha_{0} Z_{e f f}\right)^{3 / 2}}{\sqrt{V}} \exp \left(i \boldsymbol{k}_{11} \cdot \boldsymbol{r}_{e, 11}\right) \\
\cdot\left\{\begin{array}{l}
\left(\mathrm{e}^{i k_{z} z_{e}}+R \mathrm{e}^{-i k_{z} z_{e}}\right) \cdot u_{\sigma_{p}}(\tilde{0}) u_{\sigma_{e}}\left(\boldsymbol{k}_{i}\right), z<0 \\
T \mathrm{e}^{-\gamma z_{e}} \cdot u_{\sigma_{p}}(\tilde{0}) u_{\sigma_{e}}\left(\boldsymbol{k}_{i}\right), z>0 .
\end{array}\right.
\end{gathered}
$$

Here $\left(\sigma_{p}, \sigma_{e}\right)$ and $\left(\sigma_{p}^{\prime}, \sigma_{e}^{\prime}\right)$ are the spin co-ordinates in the initial and the final states of the electrons. $\left(\boldsymbol{k}_{p}, \boldsymbol{k}_{e}\right)$ and $\left(E_{p}, E_{e}\right)$ are the momenta and energies of the final two electrons and $\left(\boldsymbol{k}_{i}, E_{i}\right)$ are the momentum and energy of the incident electron. Here $u_{\sigma_{p}}(\tilde{0})$ and $u_{\sigma_{e}}\left(\boldsymbol{k}_{i}\right)$ are the Dirac spinors of the atomic elec- trons and the incident electron. Also $u_{\sigma_{p}^{\prime}}\left(\boldsymbol{k}_{p}\right)$ and $u_{\sigma_{e}^{\prime}}\left(\boldsymbol{k}_{e}\right)$ represent the Dirac spinors of scattered and ejected electron. In the Equation (23) the term

$\varphi\left(\boldsymbol{r}_{e}\right)=a \ln \left(2 k_{e, z} z_{e}\right)$ is the phase modification of the asymptotically free electron motion due to its image charge where $k_{e, z}=\hat{z} \cdot \boldsymbol{k}_{e}$ and the Sommerfeld parameter $a=-1 / 4 k_{e, z}$ indicates the strength of this interaction. In case $a \equiv 0$, we end up with the final state being a product of the two free-particles states. The final state energy is given by $E_{f}=k_{e}^{2} / 2+k_{p}^{2} / 2 m_{p}$. The refection and transmission coefficient $R$ and $T$ are given by

$$
R=\frac{\boldsymbol{k}_{z}-i \gamma}{\boldsymbol{k}_{z}+i \gamma}, T=\frac{2 \boldsymbol{k}_{z}}{\boldsymbol{k}_{z}+i \gamma}
$$

and

$$
\gamma=\sqrt{2 v_{0}-k_{z}^{2}} .
$$

In the present case, the transition amplitude term $T_{p e}^{s i n}$ may be written as in semi-relativistic integral form [23] as

$$
\begin{aligned}
& T_{p e}^{s i n}=-(2 \pi)^{-9 / 2}\left(\alpha_{0} Z_{e f f}\right)^{3 / 2} \\
& \cdot \lim _{\eta_{1} \rightarrow 0^{+}} \int \mathrm{d} \boldsymbol{r}_{p} \mathrm{~d} \boldsymbol{r}_{e} \exp \left\{-i\left[\boldsymbol{Q} \cdot \boldsymbol{r}_{p}+\boldsymbol{k}_{e} \cdot \boldsymbol{r}_{e}-\varphi\left(\boldsymbol{r}_{e}\right)\right]\right\} \\
& \times \frac{\exp \left(-\eta_{1}\left|\boldsymbol{r}_{e}-\boldsymbol{r}_{p}\right|\right)}{\left|\boldsymbol{r}_{e}-\boldsymbol{r}_{p}\right|} \chi_{k}\left(\boldsymbol{r}_{e}\right) u_{\sigma_{p}^{\prime}}^{\dagger}\left(\boldsymbol{k}_{p}\right) u_{\sigma_{p}^{\prime}}(\tilde{0}) u_{\sigma_{e}^{\prime}}^{\dagger}\left(\boldsymbol{k}_{e}\right) u_{\sigma_{e}^{\prime}}\left(\boldsymbol{k}_{i}\right) .
\end{aligned}
$$

Therefore

$$
T_{p e}^{\sin }=t_{1}\left(\boldsymbol{k}_{p}, \boldsymbol{k}_{e}\right) M_{1} M_{1}
$$

where

$$
\begin{aligned}
& t_{1}\left(\boldsymbol{k}_{p}, \boldsymbol{k}_{e}\right)=-(2 \pi)^{-9 / 2}\left(\alpha_{0} Z_{e f f}\right)^{3 / 2} \\
& \cdot \lim _{\eta_{1} \rightarrow 0^{+}} \int \mathrm{d} \boldsymbol{r}_{p} \mathrm{~d} \boldsymbol{r}_{e} \exp \left\{-i\left[\boldsymbol{Q} \cdot \boldsymbol{r}_{p}+\boldsymbol{k}_{e} \cdot \boldsymbol{r}_{e}-\varphi\left(\boldsymbol{r}_{e}\right)\right]\right\} \\
& \times \frac{\exp \left(-\eta_{1}\left|\boldsymbol{r}_{e}-\boldsymbol{r}_{p}\right|\right)}{\left|\boldsymbol{r}_{e}-\boldsymbol{r}_{p}\right|} \chi_{k}\left(\boldsymbol{r}_{e}\right)
\end{aligned}
$$

and

$$
\begin{aligned}
& M_{1}=u_{\sigma_{p}^{\prime}}^{\dagger}\left(\boldsymbol{k}_{p}\right) u_{\sigma_{p}^{\prime}}(\tilde{0}), \\
& M_{2}=u_{\sigma_{e}^{\prime}}^{\dagger}\left(\boldsymbol{k}_{e}\right) u_{\sigma_{e}^{\prime}}\left(\boldsymbol{k}_{i}\right) .
\end{aligned}
$$

Upon replacing the logarithmic phase in Equation (29) by its integral representation, given by

$$
\exp (i \varphi)=\lim _{\eta_{3} \rightarrow 0^{+}} \frac{1}{\Gamma(\alpha)} \int_{0}^{\infty} \mathrm{d} t \exp \left(-\boldsymbol{\xi} \cdot \boldsymbol{r}_{e}\right) t^{\alpha-1},
$$

where $\alpha=-i a+\eta_{3}, a=-1 / 4 k_{e, z}$ and $\xi=2 k_{e . z} t \hat{z}$. Then performing the integral over the projectile co-ordinates, Equation (29) simplifies to 


$$
\begin{aligned}
& t_{1}\left(\boldsymbol{k}_{p}, \boldsymbol{k}_{e}\right) \\
= & \frac{-\left(\alpha_{0} Z_{\text {eff }}\right)^{3 / 2}}{2 \pi^{2}} \lim _{\eta_{3}, \eta_{1} \rightarrow 0^{+}} \frac{1}{\Gamma(\alpha)\left[Q^{2}+\eta_{1}^{2}\right]} \int_{0}^{\infty} \mathrm{d} t J(t) t^{\alpha-1},
\end{aligned}
$$

where

$$
\begin{aligned}
& J(t) \\
= & (2 \pi)^{-3 / 2} \int \mathrm{d} \boldsymbol{r}_{e} \exp \left\{-i\left[\boldsymbol{Q}+\boldsymbol{k}_{e}-i \xi\right] \cdot \boldsymbol{r}_{e}\right\} \chi_{\boldsymbol{k}}\left(\boldsymbol{r}_{e}\right) \\
= & \tilde{\chi}_{k}(\bar{\lambda}, t) .
\end{aligned}
$$

In Equation (33) we introduced the complex vector $\boldsymbol{\Lambda}=\boldsymbol{Q}+\boldsymbol{k}_{e}-\boldsymbol{i} \boldsymbol{\xi}$. Using the theory [23], we have

$$
\begin{aligned}
& \tilde{\chi}_{k}(\bar{\lambda}, t) \\
= & i \sqrt{\frac{2 \pi}{V}} \delta^{2}\left(\boldsymbol{k}_{11}-\Lambda_{11}\right)\left(\frac{1}{\Lambda_{z}-k_{z}}+\frac{R}{\Lambda_{z}+k_{z}}-\frac{T}{\Lambda_{z}-i \gamma}\right),
\end{aligned}
$$

where the vector $\Lambda$ is given by $\Lambda=\boldsymbol{Q}-\boldsymbol{K}-\boldsymbol{k}_{e}-i \boldsymbol{\xi}$. By the Fourier transform $J(t)$ evaluates to

$$
\begin{aligned}
& J(t) \\
= & i \sqrt{\frac{2 \pi}{V}} \delta^{(2)}\left(\boldsymbol{k}_{11}-\Lambda_{11}\right)\left(\frac{1}{\Lambda_{z}-k_{z}}+\frac{R}{\Lambda_{z}+k_{z}}-\frac{T}{\Lambda_{z}-i \gamma}\right) .
\end{aligned}
$$

Now after inserting the Equation (35) into a Equation (32), the remaining one dimensional integral can be algebraically transformed to the Beta function integral representation

$$
\begin{aligned}
& \int_{0}^{\infty} t^{\mu-1}(1+\beta t)^{-v} \mathrm{~d} t=\beta^{-\mu} B(\mu, v-\mu) ; \\
& |\arg \beta|<\pi, R_{e}(v)>0,
\end{aligned}
$$

give

$$
\begin{aligned}
t_{1}\left(\boldsymbol{k}_{p}, \boldsymbol{k}_{e}\right) & =\frac{-\left(\alpha_{0} Z_{\text {eff }}\right)^{3 / 2}}{(2 \pi V)^{1 / 2}} \delta^{2}\left(\boldsymbol{k}_{11}-\Lambda_{11}\right) \\
& \times \lim _{\eta_{3}, \eta_{1} \rightarrow 0^{+}} \frac{1}{\Gamma(\alpha)\left[Q^{2}+\eta^{2}\right]}\left(J_{1}+R J_{2}-T J_{3}\right) .
\end{aligned}
$$

The functions $J_{i}$ are given by

$$
J_{i}=\left(\bar{b}_{i}\right)^{-1} \int_{0}^{\infty} \mathrm{d} t t^{\alpha-1}\left(1+\frac{-i 2 k_{e, z}}{\bar{b}_{i}} t\right)^{-1}, i=1,2,3,
$$

where

$$
\begin{aligned}
& \bar{b}_{1}=\bar{Q}_{z}+k_{e, z}-k_{z}, \\
& \bar{b}_{2}=\bar{Q}_{z}+k_{e, z}+k_{z}, \bar{b}_{3}=\bar{Q}_{z}+k_{e, z}-i \gamma .
\end{aligned}
$$

Making use Equation (36) and after calculation, the final expression

$$
\begin{aligned}
& t_{1}\left(\boldsymbol{k}_{p}, \boldsymbol{k}_{e}\right)=\frac{-i\left(\alpha_{0} Z_{e f f}\right)^{3 / 2}}{(2 \pi V)^{1 / 2}} \delta^{2}\left(\boldsymbol{k}_{11}-\Lambda_{11}\right) \frac{\Gamma(1+i a)}{Q^{2}} \mathrm{e}^{-2 a \pi / 3} \\
& \times \exp \left[i a \ln \left(2 k_{e, z}\right)\right] \\
& \cdot\left[\left(\bar{b}_{1}\right)^{-(1+i a)}+R \cdot\left(\bar{b}_{2}\right)^{-(1+i a)}-T \cdot\left(\bar{b}_{3}\right)^{-(1+i a)}\right] .
\end{aligned}
$$

\subsection{Analytical Calculation of the Transition Amplitude Term $T_{p, s}^{\sin }$ in Relativistic Effects}

In this case the transition amplitude term of the Equation (19), the screened Coulomb potential [26] for relativistic case is given by

$$
V^{\text {ion }}\left(\boldsymbol{r}_{p}\right)=\frac{Z_{\text {eff }} \alpha_{0}}{r_{p}} \exp \left(-\lambda_{\text {eff }} r_{p}\right) .
$$

The effective parameter $\lambda_{\text {eff }}$ account for the screaming of the pure ionic field due to the presence of the localized positive cores as well as delocalized electrons [26]. For aluminum surface the numerical value of $\lambda_{\text {eff }}$ is 0.886. Equation (19) can be written as in semi-relativistic form

$$
\begin{aligned}
T_{p, s}^{s i n}= & \left\langle\boldsymbol{k}_{p}, \boldsymbol{k}_{e}, \sigma_{p}^{\prime}, \sigma_{e}^{\prime}\left|W_{p}^{s}\right| \chi_{k}, \boldsymbol{k}_{i}, \sigma_{p}, \sigma_{e}\right\rangle \\
& +\left\langle\boldsymbol{k}_{p}, \boldsymbol{k}_{e}, \sigma_{p}^{\prime}, \sigma_{e}^{\prime}\left|W_{p e} g_{p e}^{-} W_{p}^{s}\right| \chi_{k}, \boldsymbol{k}_{i}, \sigma_{p}, \sigma_{e}\right\rangle .
\end{aligned}
$$

Here

$$
W_{p}^{s}=\sum_{i=1}^{N} V_{i}^{\text {ion }}
$$

where $V_{i}^{\text {ion }}$ is the ionic core potential at the state $i, N$ is the number of ions in the solid and $W_{p}^{s}$ is periodic in each layer parallel to the $x-y$ plane, but not in $z-$ directions. The $j$-th ion in the lth layer has the coordinates $\boldsymbol{r}_{j, l}=\left(\boldsymbol{r}_{11, j}, \boldsymbol{r}_{\perp, l}\right)$. Thus the periodic potential $W_{p}^{s}$ at the position $\boldsymbol{r}^{\prime}$ can be written as

$$
W_{p}^{s}\left(\boldsymbol{r}_{11}^{\prime}, z^{\prime}\right)=\sum_{i} \sum_{j} V^{\text {ion }}\left(\boldsymbol{r}_{11}^{\prime}-\boldsymbol{r}_{11, j}^{\prime}, z^{\prime}-r_{\perp, l}\right) .
$$

Since $W_{p}^{s}$ is periodic in the $x$ and $y$ directions, we can introduce [26] two dimensional reciprocal vectors $\boldsymbol{g}_{11}=2 \pi\left(n_{x} / d_{x}, n_{y} / d_{y}\right), n_{x}, n_{y} \in Z$ and write $W_{p}^{s}$ at the position $\boldsymbol{r}^{\prime}$ as

$$
W_{p}^{s}\left(\boldsymbol{r}_{11}^{\prime}, z^{\prime}\right)=\sum_{\bar{g}_{11}} \tilde{W}_{p}^{s}\left(\boldsymbol{g}_{11}, z^{\prime}\right) \exp \left(i \boldsymbol{g}_{11} \cdot \boldsymbol{r}_{11}^{\prime}\right) .
$$

The two dimensional $\boldsymbol{g}_{11}$ Fourier transform $\tilde{W}_{p}^{s}\left(\boldsymbol{g}_{11}, z^{\prime}\right)$ is given by

$$
\tilde{W}_{p}^{s}\left(\boldsymbol{g}_{11}, z^{\prime}\right)=\frac{1}{A_{u c}} \int_{u c} \mathrm{~d}^{2} r_{11}^{\prime} W_{p}^{s}\left(\boldsymbol{r}_{11}^{\prime}, z^{\prime}\right) \exp \left(-i \boldsymbol{g}_{11} \cdot \boldsymbol{r}_{11}^{\prime}\right)
$$

where $A_{u c}=d_{x} d_{y}$. The lattice constants in the $x, y$ and $z$ 
directions are $d_{x}, d_{y}$ and $d_{z}$ respectively.

Using Equations (23), (24) and (42) the final expression for $T_{p, s}^{s i n}$ may be written as in the semi-relativistic form

$$
T_{p, s}^{s i n}=u_{\sigma_{p}^{\prime}}^{\dagger}\left(\boldsymbol{k}_{p}\right) u_{\sigma_{p}^{\prime}}(\tilde{0}) u_{\sigma_{e}^{\prime}}^{\dagger}\left(\boldsymbol{k}_{e}\right) u_{\sigma_{e}^{\prime}}\left(\boldsymbol{k}_{i}\right) t_{2}\left(\boldsymbol{k}_{p}, \boldsymbol{k}_{e}\right),
$$

where

$$
\begin{aligned}
& t_{2}\left(\boldsymbol{k}_{p}, \boldsymbol{k}_{e}\right) \\
& =\sqrt{\frac{8}{\pi V}} \frac{\left(\alpha_{0} Z_{e f f}\right)^{5 / 2}}{A_{u c}} \mathrm{e}^{-2 a \pi / 3} \Gamma(1+i a) \exp \left[i a \ln \left(2 k_{e, z}\right)\right] \\
& \times \sum_{l, \bar{g}_{11}} \delta^{2}\left(\boldsymbol{k}_{11}-\boldsymbol{Q}_{11}+\boldsymbol{g}_{11}-\boldsymbol{k}_{e, 11}\right)\left(£_{0}+£_{1}+£_{2}\right)
\end{aligned}
$$

with

$$
\begin{aligned}
& £_{0}=\exp \left(-i z_{0} r_{\perp, l}\right) B_{0}\left[2 i\left|Q_{11}-g_{11}\right|\right]^{-1} \\
& \cdot\left[\bar{K}_{-}^{2}-\left|k_{i, 11}+\bar{g}_{11}\right|^{2}-\left(k_{i, z}+z_{0}\right)^{2}\right]^{-1} \times\left[\bar{g}_{11}^{2}+z_{0}^{2}+\lambda_{\text {eff }}^{2}\right]^{-1} .
\end{aligned}
$$

In case $\bar{K}_{-}^{2}>\left|k_{i, 11}+\bar{g}_{11}\right|^{2}$ we obtain for $£_{1}$,

$$
\begin{aligned}
£_{1}= & -(1 / 2) \exp \left(-i z_{1} r_{\perp, l}\right) B_{1}\left[\left|Q_{11}-g_{11}\right|^{2}+\left|Q_{z}-z_{1}\right|^{2}\right]^{-1} \\
& \cdot\left[\bar{K}_{-}^{2}-\left|k_{i, 11}+\bar{g}_{11}\right|^{2}\right]^{-1 / 2} \times\left[\bar{g}_{11}^{2}+z_{1}^{2}+\lambda_{\text {eff }}^{2}\right]^{-1}
\end{aligned}
$$

whereas if $\bar{K}_{-}^{2}<\left|k_{i, 11}+\bar{g}_{11}\right|^{2}$ the following relation is valid:

$$
\begin{aligned}
& £_{1}=-(i / 2) \exp \left(-i z_{1} r_{\perp, l}\right) B_{1}\left[\left|Q_{11}-g_{11}\right|^{2}+\left|Q_{z}-z_{1}\right|^{2}\right]^{-1} \\
& \cdot\left[-\bar{K}_{-}^{2}+\left|k_{i, 11}+\bar{g}_{11}\right|^{2}\right]^{-1 / 2} \times\left[\bar{g}_{11}^{2}+z_{1}^{2}+\lambda_{\text {eff }}^{2}\right]^{-1} .
\end{aligned}
$$

Also, the expression for $£_{2}$ may be written as

$$
\begin{aligned}
& £_{2}=\frac{-i}{2 \sqrt{\bar{g}_{11}^{2}+\lambda_{\text {eff }}^{2}}} \\
& \cdot \exp \left(-i z_{2} r_{\perp, l}\right) B_{2}\left[\left|Q_{11}-g_{11}\right|^{2}+\left(Q_{z}-z_{2}\right)^{2}\right]^{-1} \\
& \times\left[\bar{K}_{-}^{2}-\left|\boldsymbol{k}_{i, 11}+\bar{g}_{11}\right|^{2}-\left(k_{i, z}+z_{2}\right)^{2}\right]^{-1} .
\end{aligned}
$$

The function $B_{j} ; j=0,1,2$, we have been defined as

$$
B_{j}=\left(b_{j}-k_{z}\right)^{-(1+i a)}+R \cdot\left(b_{j}+k_{z}\right)^{-(1+i a)}-T \cdot\left(b_{j}-i \gamma\right)^{-(1+i a)}
$$

where $b_{j}=\bar{Q}_{z}-z_{j}+k_{e, z}, \quad \bar{K}_{-}=\mu_{p}\left(\boldsymbol{k}_{p} / m_{p}-\boldsymbol{k}_{e}\right)$ and $\mu_{p}=m_{p} /\left(m_{p}+1\right)$. Since $W_{p}^{\text {vac }}$ is an asymptotic (image charge ) perturbation, so the term $T_{p, v}^{s i n}$ is neglected here. Therefore the expression for the $T^{\sin }$-matrix element may be written as in semi-relativistic form

$$
T_{p, s}^{s i n}=u_{\sigma_{p}^{\prime}}^{\dagger}\left(\boldsymbol{k}_{p}\right) u_{\sigma_{p}^{\prime}}(\tilde{0}) u_{\sigma_{e}^{\prime}}^{\dagger}\left(\boldsymbol{k}_{e}\right) u_{\sigma_{e}^{\prime}}\left(\boldsymbol{k}_{i}\right) t\left(\boldsymbol{k}_{p}, \boldsymbol{k}_{e}\right)
$$

where

$$
t\left(\boldsymbol{k}_{p}, \boldsymbol{k}_{e}\right)=t_{1}\left(\boldsymbol{k}_{p}, \boldsymbol{k}_{e}\right)+t_{2}\left(\boldsymbol{k}_{p}, \boldsymbol{k}_{e}\right) .
$$

Now taking sum over final spin states and an average over initial states

$$
\begin{aligned}
& \overline{\left|T^{\sin }\right|^{2}} \\
= & \frac{1}{4} \sum\left|u_{\sigma_{p}^{\prime}}^{\dagger}\left(\boldsymbol{k}_{p}\right) u_{\sigma_{p}}(\tilde{0})\right|^{2} \sum\left|u_{\sigma_{e}^{\prime}}^{\dagger}\left(\boldsymbol{k}_{e}\right) u_{\sigma_{e}}\left(\boldsymbol{k}_{i}\right)\right|^{2}\left|t\left(\boldsymbol{k}_{p}, \boldsymbol{k}_{e}\right)\right|^{2} .
\end{aligned}
$$

Now the spin sums can be obtained

$$
\begin{gathered}
\sum\left|u_{\sigma_{p}^{\prime}}^{\dagger}\left(\boldsymbol{k}_{p}\right) u_{\sigma_{p}}(\tilde{0})\right|^{2}=\frac{\left(E_{p}+1\right)}{E_{p}}, \\
\sum\left|u_{\sigma_{e}^{\prime}}^{\dagger}\left(\boldsymbol{k}_{e}\right) u_{\sigma_{e}}\left(\boldsymbol{k}_{i}\right)\right|^{2}=\frac{\left(E_{i} E_{p}+1+\boldsymbol{k}_{i} \cdot \boldsymbol{k}_{e}\right)}{E_{p} E_{e}} .
\end{gathered}
$$

So, we obtain from Equation (56),

$$
\begin{aligned}
& \overline{\left|T^{\sin }\right|^{2}} \\
= & \left(E_{i} E_{e}+1+\boldsymbol{k}_{i} \cdot \boldsymbol{k}_{e}\right)\left(E_{p}+1\right) \frac{\left[t_{R}^{2}\left(\boldsymbol{k}_{p}, \boldsymbol{k}_{e}\right)+t_{I}^{2}\left(\boldsymbol{k}_{p}, \boldsymbol{k}_{e}\right)\right]}{E_{p} E_{e} E_{i}}
\end{aligned}
$$

where $t_{R}\left(\boldsymbol{k}_{p}, \boldsymbol{k}_{e}\right)$ and $t_{I}\left(\boldsymbol{k}_{p}, \boldsymbol{k}_{e}\right)$ are the real and imaginary parts of the $T^{\sin }$ matrix element.

Finally, the differential cross-section [23] for $T^{\text {sin }}$ matrix in semi-relativistic form is given by

$$
\frac{\mathrm{d} \sigma\left(\boldsymbol{k}_{i}, \boldsymbol{k}\right)}{\mathrm{d} \Omega_{e} \mathrm{~d} E_{e} \mathrm{~d}^{2} \Omega_{p} \mathrm{~d} E_{p}}=(2 \pi)^{4} \frac{k_{p} k_{e}}{v_{i}} \overline{\left.T^{\sin }\right|^{2}}\left(E_{f}-E_{i}\right)
$$

where $\quad v_{i}=\left|\boldsymbol{k}_{i}\right| / m_{p}, \quad E_{f}=k_{p}^{2} / 2 m_{p}-k_{e}^{2} / 2 \quad$ also $\Omega_{e}$ and $\Omega_{p}$ are the solid angles of the emitted electron and scattered projectile respectively.

This is the modified formal theory on semi-relativistic effects during electron emission from metallic surfaces upon impact of high energy particles to determine differential cross-section of different kinematic conditions.

\section{Concluding Remarks}

A theoretical formulation of scattering cross-section of high energy change particles from clean metallic semiinfinite solid in the relativistic region has presented single scattering amplitude $T^{\text {sin }}$ term [23]. In this theory, for multiply differential cross-sections, we used a semi-relativistic Jellium initial state wave function. A relativistic final state electron surface image charge interaction has been included in its asymptotic form. We will utilize another multiple scattering term $T^{\text {mult }}$

[23] 
which can provide better results for cross-section. Such analytical calculation and numerical results are in progress and will be reported in the next work.

\section{Acknowledgements}

Part of this work was done when one of the authors (SD) was the DAAD Fellow at the institute of Physics, Martin-Luther University, Halle, Germany under Prof. Jamal Berakdar. This work also partially supported by Higher Education Quality Enhancement Program (HEQEP) of World Bank.

\section{REFERENCES}

[1] J. N. Das, "Differential Cross-Section for the Inner-Shell Ionization of Medium-Heavy Atoms by the Electrons," Il Nuovo Cimento B, Vol. 12, No. 2, 1972, pp. 197-204. http://dx.doi.org/10.1007/BF02822628

[2] J. N. Das and S. Chakraborthy, "Atomic Inner-Shell Ionization," Physical Review A, Vol. 32, No. 1, 1985, pp. 176-180. http://dx.doi.org/10.1103/PhysRevA.32.176

[3] D. H. Jakubassa-Amundsen, "A Systematic Study of Relativistic (e, 2e) Collisions in Comparison with Experiment," Journal of Physics B: Atomic, Molecular and Optical Physics, Vol. 25, No. 6, 1992, p. 1297. http://dx.doi.org/10.1088/0953-4075/25/6/020

[4] X. Zhang, C. T. Whelan and H. R. J. Walters, "Local Supersymmetry for Spinning Particles," Physics Letters B, Vol. 64, No. 4, 1976, pp. 435-438. http://dx.doi.org/10.1016/0370-2693(76)90115-5

[5] Allan, P. Bickert and W. Hink, "(e, 2e) Cross Sections for Inner-Shell Ionization of Argon and Neon," Journal of Physics B: Atomic, Molecular and Optical Physics, Vol. 25, No. 20, 1992, p. 4325. http://dx.doi.org/10.1088/0953-4075/25/20/025

[6] S. Kellir, R. M. Dreizler. L. U. Ancarni and H. R. J. Walfers, Z. Phys. D, Vol. 37, 1996, p. 191. http://dx.doi.org/10.1007/s004600050028

[7] E. Schüle and W. Nakel, "Triply-Differential Cross Section for K-Shell Ionisation Of silver by Relativistic Electron Impact," Journal of Physics B: Atomic, Molecular and Optical Physics, Vol. 15, No. 17, 1982, p. L639. http://dx.doi.org/10.1088/0022-3700/15/17/013

[8] H. Ruoff and W. Nakel, "Absolute Triply Differential Cross Section for K-Shell Ionisation by Relativistic Electron Impact for High Atomic Number," Journal of Physics B: Atomic, Molecular and Optical Physics, Vol. 20, No. 10, 1987, p. 2299. http://dx.doi.org/10.1088/0022-3700/20/10/022

[9] J. Bonfert, H. Graf and W. Nakel, "Relativistic (e,2e) Processes on Atomic Inner Shells," Journal of Physics B: Atomic, Molecular and Optical Physics, Vol. 24, No. 6, 1991, p. 1423.

http://dx.doi.org/10.1088/0953-4075/24/6/026

[10] J. N. Das and S. Dhar, "Energy Spectrum of Scattered Electrons in K-Shell Ionization of Medium to Heavy Atoms by Relativistic Electrons," Journal of Physics B:
Atomic, Molecular and Optical Physics, Vol. 31, No. 10, 1998, p. 2355.

http://dx.doi.org/10.1088/0953-4075/31/10/021

[11] S. Dhar and M. R. Alam, "Computation of Triple Differential Cross-Sections with the Inclusion of Exchange Effects in Atomic K-Shell Ionization by Relativistic Electrons for Symmetric Geometry," Pramana, Vol. 69, No. 3, 2007, p. 387. http://dx.doi.org/10.1007/s12043-007-0140-3

[12] S. Dhar, "The Energy Spectrum of Scattered Particles in the K-Shell Ionization of Medium Heavy Atoms by Relativistic Electrons and Positrons with Exchange Effects," Journal of Physics B: Atomic, Molecular and Optical Physics, Vol. 41, No. 15, 2008, p. 155204. http://dx.doi.org/10.1088/0953-4075/41/15/155204

[13] I. E. Mc Carthy and E. Weigold, "Electron Momentum Spectroscopy of Atoms and Molecules," Reports on Progress in Physics, Vol. 54, No. 6, 1991, p. 789. http://dx.doi.org/10.1088/0034-4885/54/6/001

[14] H. Ehrhardt, K. Jung, G. Knoth and P. Schlemmer, "Differential Cross Sections of Direct Single Electron Impact Ionization," Zeitschrift für Physik D Atoms, Molecules and Clusters, Vol. 1, No. 1, 1986, pp. 3-32. http://dx.doi.org/10.1007/BF01384654

[15] F. W. Byron Jr. and C. J. Joachain, "Theory of (e, 2e) Reactions," Physics Reports, Vol. 179, No. 4, 1989, pp. 211-272. http://dx.doi.org/10.1016/0370-1573(89)90158-0

[16] C. T. Whelan, R. J. Allan, J. Rasch, H. R. J. Walters, X. Zhang, J. Roder, K. Jung and H. Ehrhardt, "Coulomb three-Body Effects in (e,2e) Collisions: The Ionization of H in Coplanar Symmetric Geometry," Physical Review A, Vol. 50, No. 5, 1994, pp. 4394-4396. http://dx.doi.org/10.1103/PhysRevA.50.4394

[17] J. N. Das and S. Seal, "Electron-Hydrogenatom Ionization Collisions at Intermediate (5I0-20I0) and High $(\gtrless$ 20I0) Energies," Physical Review A, Vol. 47, No. 4, 1993, pp. 2978-2986. http://dx.doi.org/10.1103/PhysRevA.47.2978

[18] J. N. Das and S. Seal, Australian Journal of Physics, Vol. 47, 1994, p. 99.

[19] Sunil Dhar, "Electron Impact Ionisation of Metastable 2s-state Hydrogen Atoms," Australian Journal of Physics, Vol. 47, No. 5, 1994, pp. 937-944. http://dx.doi.org/10.1071/PH960937

[20] J. N.Das and S. Dhar, "Energy Spectrum of Ejected Electrons in Ionization of Hydrogen Atoms by Electrons," Pramana, Vol. 53, No. 5, 1999, pp. 869-875. http://dx.doi.org/10.1007/s12043-999-0121-9

[21] J. Berakdar, J. Roder, J. S. Brggs and H. Ehrhardt, "The Effect of Target Atom Polarization and Wavefunction Distortion in (e - 2e) Ionization of Hydrogen," Journal of Physics B: Atomic, Molecular and Optical Physics, Vol. 29, No. 9, 1996, p. 1767. http://dx.doi.org/10.1088/0953-4075/29/9/021

[22] P. J. Jenning, R. O. Jones, and M. Weinert, "Surface Barrier for Electrons in Metals," Physical Review B, Vol. 37, No. 11, 1988, pp. 6113-6120. http://dx.doi.org/10.1103/PhysRevB.37.6113 
[23] J. Berakdar and M. P. Das, "Electron Ejection from Clean Metallic Surfaces upon Charged Particle Impact," Physical Review A, Vol. 56, No. 2, 1997, pp. 1403-1413. http://dx.doi.org/10.1103/PhysRevA.56.1403

[24] C. B. Duke, "Advances in Chemical Physies," Wiley, New York, 1974.
[25] J. B. Pendry, "Low Energy Electron Diffraction," Academic, London, 1974.

[26] U. Thumm and J. S. Briggs, Nuclear Instruments and Methods in Physics Research Section B, Vol. 43, 1989, p. 471. 